\section{La fièvre récurrente du paludisme: un atout majeur pour le parasite}

Dominique Labie
Inserm U.567, Institut

Cochin, 24, rue du Faubourg

Saint-Jacques, 75014 Paris,

France.

labie@cochin.inserm.fr

indiens de Bangalore a étudié le mécanisme moléculaire mis en jeu dans cette action promotrice des accès fébriles sur le développement du Plasmodium [3]. Dans un travail antérieur, les mêmes auteurs avaient focalisé leur attention sur le rôle d'une protéine de choc thermique dans la croissance et le développement du parasite [4]. Ils se fondaient sur le rôle connu de la protéine $\mathrm{Hsp} 90$, en association avec d'autres protéines, chez les eucaryotes. Hsp90 est un chaperon impliqué dans les repliements conformationnels, mais contrôle aussi l'activité de facteurs de transcription et de protéine kinases. Comme chez les mammifères, la protéine homologue de Hsp90, PfHsp90, est incluse dans un complexe chaperon multiprotéique, dissocié par l'action de la geldamycine [4]. Les protéines de ce complexe ont pu être identifiées (lyse douce, suivie d'une immunoprécipitation par des anticorps contre PfHsp90, et électrophorèse bidimensionnelle). Les auteurs ont, comme cela était attendu, retrouvé un dimère de PfHsp90, la protéine PfHsp70, mais aussi deux autres protéines qui se sont révélées être (après digestion trypsique en gel) une protéine phosphatase 5 et une tubuline, dont l'interaction avec Hsp90 avait déjà été observée chez les mammifères $[5,6]$.

Chez Plasmodium, PfHsp90 intervenaitelle dans le cycle du parasite? On a pu montrer une inhibition de la croissance du parasite par un antibiotique de la famille des benzoquinones, la geldamy- 
cine, en même temps qu'un site potentiel de fixation de cet antibiotique sur l'extrémité aminoterminale de PfHsp90. Une étude cristallographique antérieure, déterminant la structure d'un complexe Hsp90-geldamycine corroborait cette hypothèse [7]. Restait à préciser le rapport entre le rôle de PsHsp90 et le cycle de température du paludisme. Les auteurs ont reproduit en culture (voir le protocole sur la Figure 1) l'équivalent des épisodes fébriles observés à intervalles réguliers chez les malades: deux chocs thermiques à $40^{\circ} \mathrm{C}$, séparés par un intervalle de 10 heures à $37^{\circ} \mathrm{C}$, avaientils une influence sur la croissance du parasite?

Dans les cultures témoins n'ayant pas subi le premier choc thermique, une chute massive du nombre de parasites est observée au cours du $2^{\mathrm{e}}$ choc thermique (de $\sim 12 \quad 000 / 100 \mu \mathrm{l}$ à $\sim 2000 / 100 \mu \mathrm{l})$ et le stade trophozoïte n'apparaît qu'à la fin de ce $2^{\mathrm{e}}$ choc thermique (12 heures). Aucune diminution, en revanche, n'est observée quand il y a eu le $1^{\text {er }}$ choc thermique et l'évolution vers le stade trophozoïte est nette dès la sixième heure. Ces résultats suggèrent que le parasite est en quelque sorte « protégé » contre l'action de l'élévation de température. La question suivante était de savoir si cette transition accélérée avait pour conséquence un cycle infectieux plus efficace? Après le $2^{e}$ choc thermique, les cultures ont été ramenées à $37^{\circ} \mathrm{C}$ pour 12 heures; l'augmentation du nombre de nouveaux parasites au stade anneau est deux fois supérieure quand il y a eu deux chocs thermiques successifs et non un seul, les épisodes fébriles «accélérant »le développement intra-érythrocytaire du parasite. II n'est pas sans intérêt de noter que cette régulation du développement d'un parasite par la fièvre, et donc par un choc thermique, a déjà été observée chez Trypanosoma et chez Leishmania [8], mais n'avait pas été encore décrite pour Plasmodium. Une dernière étape de cette étude a été de comprendre le rôle des protéines de choc thermique en utilisant la geldamycine, inhibiteur spécifique de Hsp90, introduite dans les cultures au début du $2^{\mathrm{e}}$ choc thermique. La présence de l'antibiotique interfère avec une transition accélérée et se traduit par un retard du passage au stade trophozoïte, qui n'est observé qu'après 12 heures au lieu de 6. Cette observation confirme donc le rôle de PfHsp90 dans cette étape du cycle parasitaire qui n'est, cependant, pas inhibée, mais seulement retardée. Des résultats similaires ont été obtenus quand la phase B est étendue à 48 heures, simulant au mieux le paludisme à $P$. falciparum.

Le complexe chaperon multiprotéique incluant la PfHsp90 semble donc nécessaire pour «protéger » le parasite contre les élévations brutales de température. La spécificité clinique du paludisme - la fièvre récurrente à intervalles réguliers est ainsi démontrée comme fondamentale dans son mécanisme pathogénique, puisque cette fièvre même stimule la croissance et le développement du Plasmodium. Les mécanismes en jeu, impliquant l'intégrité du complexe d'une protéine de choc thermique PfHsp90, pourront-ils suggérer un abord thérapeutique inédit? Le besoin s'en fait sentir toujours davantage. $\diamond$

Recurrent fever in malaria:

a main advantage

for Plasmodium falciparum?

\section{RÉFÉRENCES}

1. Kwiatkowski D. Febrile temperature can synchronize the growth of Plasmodium falciparum in vitro. J Exp Med 1996; 169: 357-61.

2. Udomsangpetch R, Pipitaporn B, Silamut K, et al. Febrile temperatures induce cytoadherence of ring-stage Plasmodium falciparum-infected erythrocytes. Proc Natl Acad Sci USA 2002; 99: 11825-9.

3. Pavithra SR, Banumathy G, Joy 0, et al. Recurrent fever promotes Plasmodium falciparum development in human erythrocytes. J Biol Chem 2004; 279 : 46692-9.

4. Banumathy G, Singh V, Pavithra SR, Tatu U. Heat shock protein 90 function is essential for Plasmodium falciparum growth in human erythrocytes. J Biol Chem 2003; 278: 18336-45.

5. Shao J, Hartson SD, Matts RL. Evidence that protein phosphatase 5 functions to negatively modulate the maturation of the Hsp90-dependent heme-regulated elF2 $\alpha$ kinase. Biochemistry 2002;

41: 6770-9.

6. Garnier C, Barbier P, Gilli R, et al. Heat-shock protein 90 (hsp90) binds in vitro to tubulin dimer and inhibits microtubule formation. Biochem Biophys Res Commun 1998; 250: 414-9.

7. Stebbins C $\varepsilon$, Russo AA, Schneider C, et al. Crystal structure of an $\mathrm{Hsp} 90$-geldanamycin complex: targeting of a protein chaperone by an antitumor agent. Cell 1997; 89: 239-50.

8. Wiesgigl M, Clos J. Heat shock protein 90 homeostasis controls stage differentiation in Leishmania donovani. Mol Biol Cell 2001; 12: 3307-16.

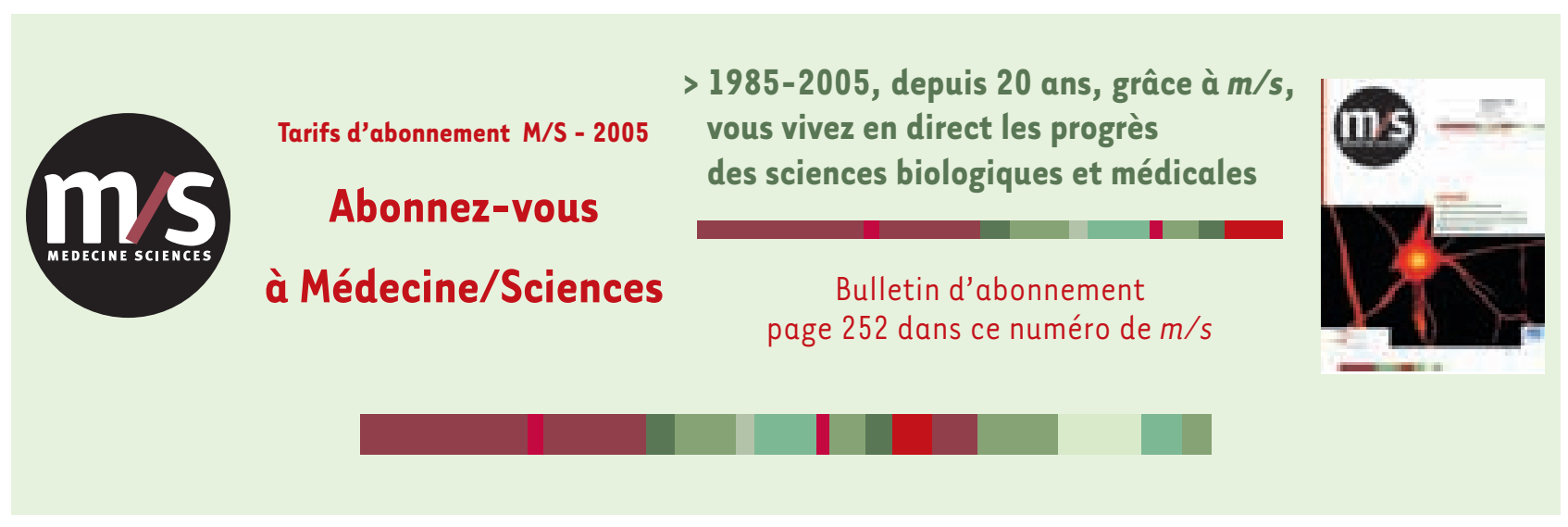

